\title{
REDES DE REUSO DE ÁGUAS INDUSTRIAIS II: ESTADO DA ARTE
}

Leonardo Oliveira Santos de Santana ${ }^{1}$, Edna dos Santos Almeida ${ }^{1}$, Kátia Katia Goés Macedo de Oliveira ${ }^{1}$

Centro universitário SENAI-CIMATEC; Salvador/Ba; leosantana049@gmail.com

Resumo: Este artigo trata da temática de redes de reuso de águas industriais. A partir de uma revisão sistemática sobre redes de reuso de água industriais objetivou-se trazer um panorama geral do que está sendo discutido e pesquisado sobre este tema e uma avaliação dos dados quantitativos das pesquisas geradas na área. A metodologia empregada foi a realização de uma revisão sistemática e análise de artigos a partir de palavras-chave. Os trabalhos relatam estudos sobre os aspectos devem levados em conta na implementação dessas redes de reuso, tais custo de tratamento para sistemas com contaminante único ou multicontaminante, topologia local, tratamento centralizado e descentralizado e os eco parques industriais. Por fim, foi feita uma síntese sobre as pesquisas realizadas na área das redes de reuso de água.

Palavras-Chave: Redes de reuso de água, tratamento de efluentes, efluentes industriais, otimização.

\section{INDUSTRIAL WASTEWATER REUSE NETWORKS II: STATE OF ART}

Abstract: This article deals with the technique of industrial water reuse networks. From a systematic review of working water networks, the objective is to provide an overview of research and research on the subject and an evaluation of the quantitative data from research generated in the area. The journal used for conducting a systematic review and analysis of articles is one from keywords. Information systems related work in containment systems can be used as control systems for single or multicontaminated contamination, local topology, centralized and decentralized treatment and industrial ecosystems. Finally, a survey was conducted on the areas of water networks.

Keywords: Water reuse networks, wastewater treatment, industrial effluents, optimization. 


\section{INTRODUÇÃO}

O uso intensivo de água nas indústrias e a visão fim de tubo que por anos prevaleceram nos modelos de produção, resultando na poluição do meio ambiente e na escassez de água em algumas regiões ${ }^{[1]}$, dão espaço hoje à busca por soluções sustentáveis, que implicam em um sistema de produção mais limpo, ao mesmo tempo que continuam trazendo lucros e que tenham relevância socioambiental ${ }^{[2]}$. Além disso as indústrias são instigadas a fazer cada vez mais o uso consciente da água em seus processos produtivos devido às regulamentações ambientais cada vez mais rigorosas sobre a descarga de águas residuais e implementação de gestão ambiental nos processos produtivos de acordo com as normas ISO 14000, em função dos impactos a serem causados nos ecossistemas naturais ${ }^{[3]}$.

Segundo Mierzwa e Hespanhol [1] e Fadzil [4] as atividades industriais são a segunda atividade humana que mais consome água, ficando atrás apenas da irrigação. Neste contexto, o gerenciamento de recursos hídricos se tornou essencial na busca por processos otimizados que promovam conservação e uso racional da água [5]. Dessa forma, uma das estratégias mais aplicadas é a implementação de redes de reuso de águas residuais. ${ }^{[3,6]}$ que tem por finalidade o aumento na eficiência na utilização de água, reduzindo ao máximo o descarte e a perda de águas e efluentes nas indústrias, conservando os ecossistemas e reduzindo e prevenindo a poluição.

Para a implementação de uma rede de reuso um dos maiores desafios é a identificação das ofertas e demandas por água de reuso. Conhecer previamente o modo de distribuição, o público que se pretende atender, assim como as vazões de entrada e saída, são etapas imprescindíveis para a elaboração de estudos preliminares e posterior implementação de uma rede de reuso ${ }^{[7]}$, dessa forma, projetos de redes de regeneração e reutilização de efluentes que tenham boa relação custobenefício tem sido o foco de muitos estudos, principalmente estudos que utilizam técnicas de otimização matemática com auxílio de simulação computacional ${ }^{[3,6]}$.

Neste contexto, este trabalho tem por objetivo trazer, a partir da revisão sistemática de publicações acerca do tema, informações sobre o estado da arte, o que está sendo pesquisado, o que deve ser levado em conta e os aspectos relevantes na implantação de uma rede de reuso de água.

\section{METODOLOGIA}

A metodologia aplicada nesse trabalho envolveu a realização de buscas nas bases de pesquisa Web of Science, Scielo, Google acadêmico e Scopus. Foram utilizadas a combinação das palavras-chave: "Reuse network near industrial", "industrial water reuse network and optimization and effluent or wastewater", "nonlinear program and industrial effluent or wastewater and treatment", "reuse network and analysis of industrial effluent", "reuse near industrial and methods of treatment and industrial effluent or wastewater", "industrial effluent treatment plant and methods of treatment and optimization", entre outras.

O período de busca foi delimitado em 10 anos, e foram aplicados os critérios de exclusão: publicações que não tinham o artigo completo disponível, de acordo com a aderência aos objetivos da pesquisa e duplicidade nas diferentes bases de pesquisa. 


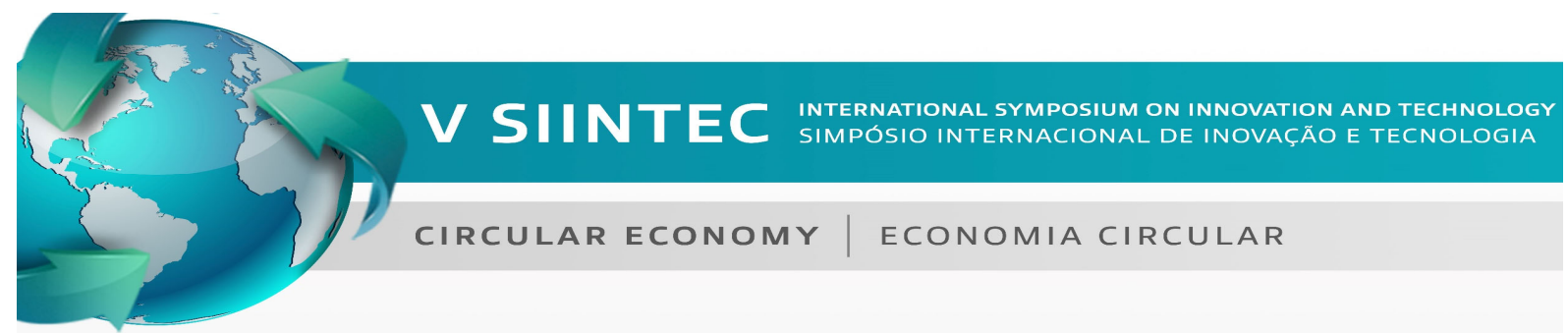

A partir da pesquisa realizada nas plataformas foi elaborada uma planilha no Microsoft Excel囚 e as publicações encontradas foram ordenadas e agrupadas.

Os artigos recuperados foram classificados de acordo com os critérios: Artigos sobre redes de reuso de águas; artigos sobre tratamento de efluentes (incluindo a otimização das ETEs) ; temática diversa relacionada a reuso de água (Produção mais limpa, otimização de processos, etc.).

O enfoque principal desse trabalho está nas redes de reuso. A leitura dos artigos encontrados propiciou o levantamento e a identificação dos aspectos de construção de uma rede de reuso, assim como dos das novidades acerca da temática. A partir dos trabalhos encontrados pôde-se então executar este artigo com um apanhado geral do estado da arte do tema.

\section{RESULTADOS E DISCUSSÃO}

A Revisão sistemática identificou um crescimento da produção cientifica sobre o tema (figura 1), com um número total de 49 artigos (Figura 2). A Figura 2 mostra também a classificação dos artigos de acordo com os critérios adotados, que gerou 3 linhas principais de subtemas ${ }^{[8]}$.

Figura 1: Números de artigos baixados por ano de publicação.

Figura 2: Artigos encontrados na revisão

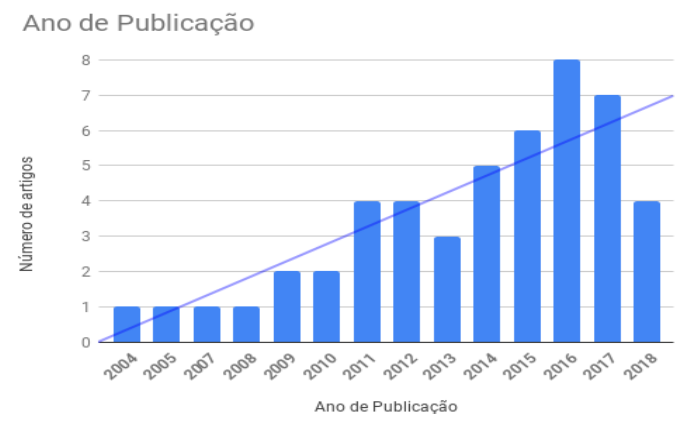

Fonte: Própria, 2018.

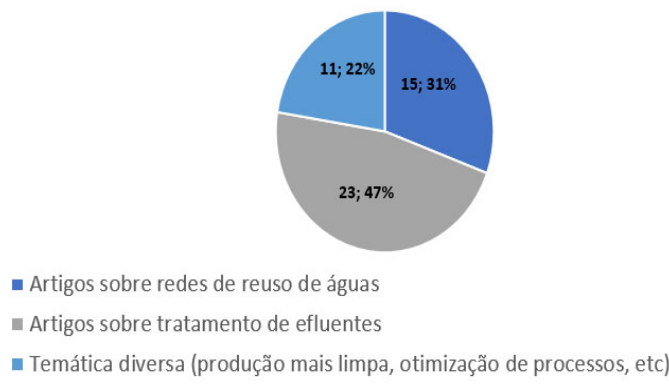

Fonte: Própria,2019

Três trabalhos de Alnouri et al ${ }^{[3,6,10]}$, estudaram a implantação de redes de reuso, entre unidades dentro de uma única planta, a viabilidade de integração entre plantas, em parques industriais, o que também foi estudado por Tan et al [8], além disso os artigos estudaram a possibilidade de implantação de uma única linha para coleta dos efluentes e a topologia local das instalações industriais. ${ }^{[3,6,9]}$

Segundo Alnouri, et al [6] planejamento de uma rede de reutilização de águas residuais deve ter enfoque nos seguintes aspectos: I ) na contabilização dos locais e na distribuição espacial de todas as entidades localizadas dentro de uma zona industrial; (II) na capacidade de incorporar informações de layout associadas a instalações de processamento envolvendo uso ou produção de água; (III) deve-se coletar informações relacionadas à presença de infraestrutura urbana comum, como a existência de corredores industriais utilizados para o transporte de água. Os aspectos espaciais para redes de regeneração e reutilização de efluentes também tendo sido estudado em outro trabalho de Alnouri et al. ${ }^{[6,3]}$

Após a sondagem dos aspectos supracitados, deve haver a seleção de tecnologias de tratamento apropriadas, bem como a colocação eficiente das instalações de tratamento correspondentes. Neste sentido dois pontos importantes 


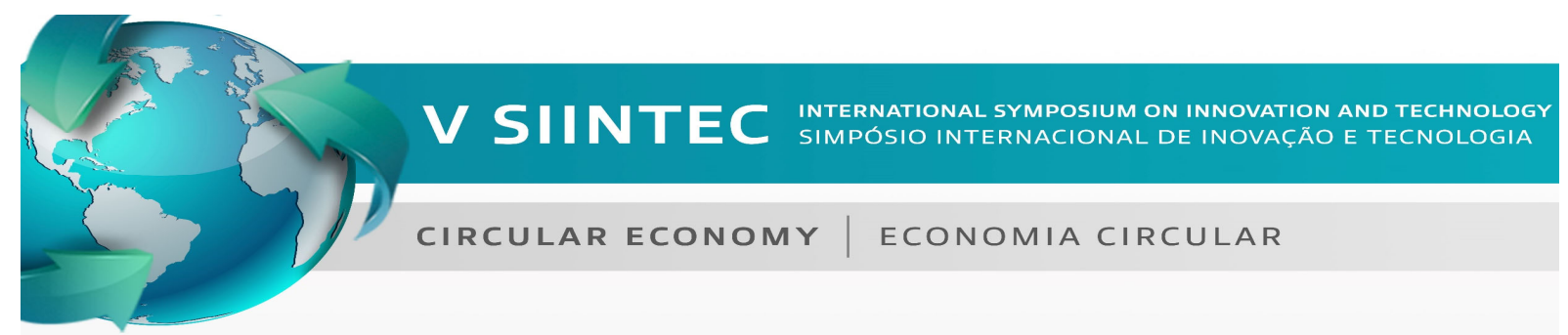

foram reportados: (1) um grupo de estabelecimentos de processamento compartilhando uma instalação de tratamento comum (centralizada), (2) a colocação de uma instalação de tratamento como uma entidade individual pertencente a um determinado local industrial (descentralizado). ${ }^{[6]}$

A seguir, estão descritos os dados importantes para avaliar uma rede de reuso.

3.1- Topologia local e redes ótimas de reuso (informações de relevo e barreiras)

Um aspecto importante a ser levado em conta no projeto de uma rede de reuso são os espaciais dentro de zonas industriais, uma vez respeitadas as barreiras físicas do sistema (Figura 02) e contabilizados os corredores da cidade industrial que são alocados para o transporte de água temos metodologias que podem ser aplicadas a problemas envolvendo qualquer tipo de estratégias de integração de água. Com representação flexível, permitindo a integração eficaz da água e a correspondência entre os fluxos de água disponíveis usando uma abordagem espacialmente restrita que utiliza as opções de caminho mais curto disponíveis. ${ }^{[10]}$

A representação e desenvolvimento de uma boa rede de reuso deve considerar os corredores, as portas de acesso, assim como as áreas obstruídas, dessa forma podese obter os caminhos mais curtos que permitirão o mapeamento adequado de origem e destino da água (fonte e sumidouro). Alnouri et al [10], utilizaram o algoritmo de Dijkistra para extrair todas as distâncias mais curtas, dado um conjunto de fontes e destinos de água dentro da planta. ${ }^{[10]}$

É possível inferir que a rede ideal estará sujeita à configuração física da planta, ou seja, o método matemático deve contemplar as regiões inativas, o que dará uma delimitação quanto ao melhor caminho, mas produzirá resultados factíveis de serem executados, sem a geração de custos com o redesenho da planta. Fazer mudanças nas configurações físicas da planta em si, só serão admitidas em último caso, se não houver outra maneira de desenvolver a rede de reuso e se os custos compensarem. [10]

Além disso fatores como a altura relativa das fontes e sumidouros de água devem ser levadas em conta. Uma vez que o bombeamento consome grande parte da energia em uma planta ou em uma rede de distribuição. Encontrar a distribuição ótima pode reduzir os custos com bombeamento e tornar viável a implantação de uma rede. ${ }^{[10]}$

Figura 02: Regiões ativas e inativas para implementação de uma rede de reuso.

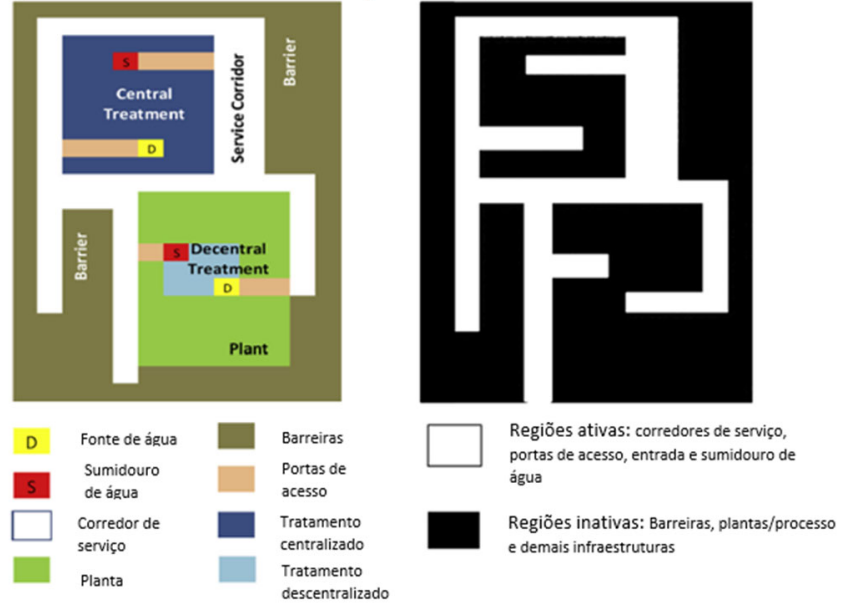

Fonte: Alnouri, 2015 (adaptado). 


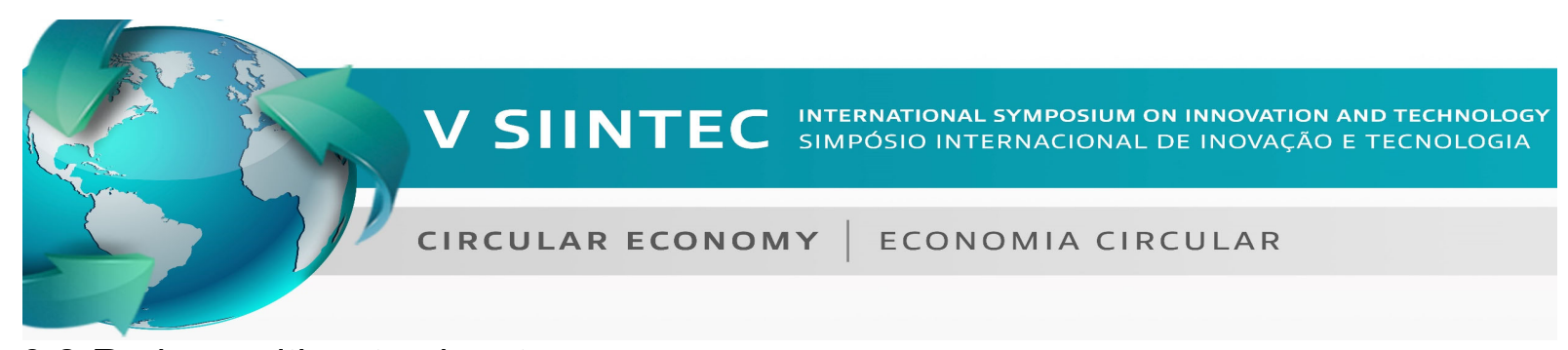

\section{2-Redes multicontaminantes}

Além disso, nas primeiras metodologias de redes de reuso, os projetos estavam limitados a redes envolvendo apenas um único contaminante. Mais tarde, os esforços de pesquisa foram estendidos para lidar com vários contaminantes. Alguns trabalhos otimizaram problemas de rede de água envolvendo múltiplos contaminantes através da combinação de técnicas de análise de water-pinch com ferramentas de programação matemática. ${ }^{[10]}$

Wang e Smith [12] propuseram uma metodologia teórica que visa maximizar a reutilização de água nas indústrias de processo para contaminantes únicos e múltiplos, nos quais restrições como a força motriz mínima de transferência de massa, incrustações e limitações de corrosão podem ser especificadas. Além disso, oportunidades de regeneração de água também foram identificadas através de uma fase de focalização. ${ }^{[3]}$

Fatores que afetam a reutilização da água recuperada e a análise exautiva e sistemática do desenho da planta são a base para se iniciar o procedimento de reavaliação e reconfiguração da planta. O uso de um modelo matemático para analisar a extensão em que os efluentes deveriam ser recuperados para uso industrial são amplamente utilizados como metodologia. ${ }^{[3,11]}$

Segundo $\mathrm{Li}^{[13]}$, a mistura desnecessária de correntes com diferentes concentrações de contaminantes deve ser minimizada, uma vez que, correntes pouco concentradas demandam menos custo de tratamento, se por um lado, a mistura de correntes diminui a concentração da corrente mais contaminada, a corrente menos "suja" demandará mais custo (Figura 03), inviabilizando o custo benefício da rede.

Figura 03: Custo de uma rede de reuso otimizada para contaminante único ou multicontaminante

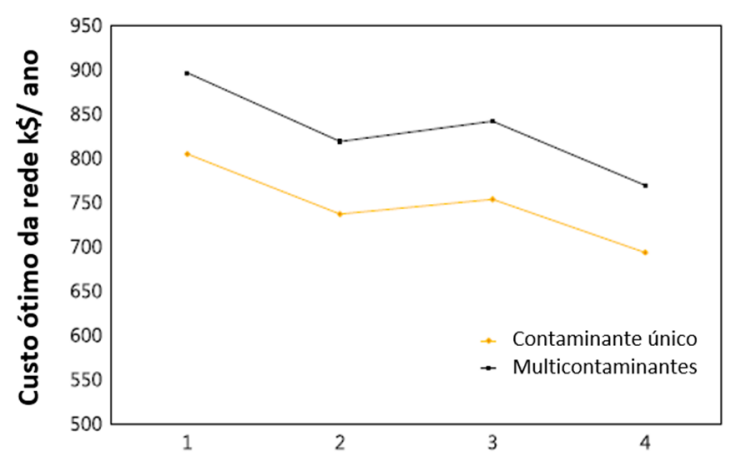

Fonte: Alnouri, 2014 (adaptado)

\section{3- Tratamento centralizado e descentralizado}

Pode-se contar com duas abordagens de otimização para o desenvolvimento de redes macroscópicas para integração de água em cidades industriais. A metodologia considera várias estratégias para a reutilização de águas residuais industriais entre diferentes instalações de processamento que operam dentro da cidade. As duas abordagens são: 1) tratamento de água "descentralizado", no local dentro de cada planta, e 2) tratamento de água "centralizado", fora do local que pode ser compartilhado entre um grupo de plantas industriais (Figura 4). O objetivo geral é desenvolver redes de água eficientes em termos de custos que possam atingir 
cenários eficazes de integração hídrica entre plantas, reduzindo consideravelmente o consumo de água doce e a descarga de águas residuais. Esse projeto de rede de água também tem sido aplicado a problemas envolvendo os chamados parques eco industriais (conjuntos industrias proximas que compartilham recursos de maneira eficiente). ${ }^{[10]}$

Figura 04: Opções de tratamento de efluente centralizado e descentralizado.

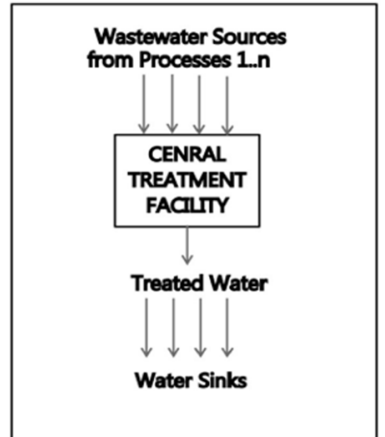

(a). Off-site treatment

Central de tratamento de efluentes

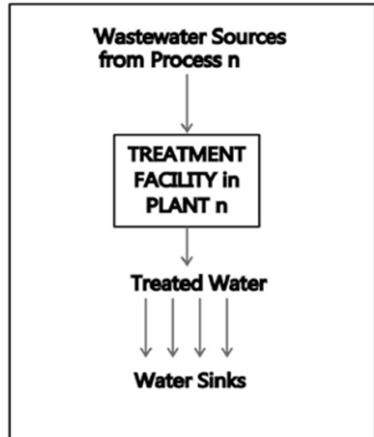

(b). On-site treatment

Tratamento descentralizado (dentro da planta)

Fonte: ALNOURI, 2015 (adaptado).

\section{4- Eco parques industriais}

Eco parques industriais são conjuntos de processos que compartilham recursos comuns de maneira eficiente, podendo haver a criação conjunta de unidades industriais para recuperação de recursos e possíveis estratégias para a troca de recursos e subprodutos, entre um grupo de industrias vizinhas. Já no fim dos anos 1980, o conceito de sintetizar redes de troca de massa, bem como o desenvolvimento de ferramentas sistemáticas para seu design ideal[ ${ }^{[3,10]}$. Logo em seguida, o desenho de redes de troca de água em eco parques industriais (EPIs) tornou-se tema de muitas contribuições de pesquisa. Vários métodos de integração de processos, tais como programação matemática e análise Pinch, já citados, têm sido utilizados para o projeto de redes de troca de água em EPIs. Diversos trabalhos exploraram os métodos de integração energética para minimizar o uso de água, sem necessariamente reutilizar efluentes, e para fazer a minimização energética; ou para gerar redes ótimas de reuso de água entre industrias, utilizando principalmente os métodos matemáticos MILP e MINLP. [10]

\section{5- Fusão de tubulações}

Por fim Alnouri et al ${ }^{[5]}$ vai trabalhar com a possibilidade de utilização de uma única linha. A maioria dos problemas de integração de água Inter plantados associaram cada alocação de água a um duto separado e o autor então apresentou uma estratégia de fusão e montagem de gasodutos para redes de regeneração e reutilização de águas residuais. Neste mesmo trabalho o autor apresentou novamente a abordagem de tratamento centralizado e descentralizado.

De modo geral, a construção de redes de reuso contendo coleta e transmissão de água entre plantas requer disponibilidade de infraestrutura, geralmente entre um 
grupo de fábricas ou plantas de uma mesma industria, dentro da proximidade geográfica, dessa forma, é sempre considerado mais econômico empregar uma transmissão de tubo único em vez de múltiplos tubos paralelos. Mesclar segmentos de dutos comuns que possuem qualidades de água semelhantes permite melhorias de custo no projeto da rede, além de reduzir a complexidade geral da rede de dutos devido à menor necessidade de interconexão de dutos. ${ }^{[6]}$

O autor desenvolveu duas metodologias:

a) Esquema de ramificação para frente: Nesse caso temos a transmissão de água de um local comum para vários destinos próximos. As linhas são ramificadas a partir de um tubo grande em várias linhas de diâmetro menor. Essas serão direcionadas para os destinos onde serão utilizadas, seguindo o esquemático da Figura 5.

b) Esquema de ramificação para trás: Essa abordagem corresponde à transmissão de água de vários locais próximos para um único destino. Várias linhas de diâmetro pequeno irão "desaguar" em uma linha de diâmetro maior, que levará a água até o ponto de consumo.

É importante notar que a mistura dependerá da composição do efluente, sendo recomendado que apenas efluentes com características físico-químicas próximas (contaminantes iguais ou quase iguais) e com concentrações próximas sejam misturados em uma única linha, esse método reduz custo para implantação da rede, uma vez que no lugar de várias tubulações se coloca apenas uma um pouco maior, mas ainda apresenta restrições.

Figura 05: Esquema de uma rede de reuso genérica com fusão das tubulações.

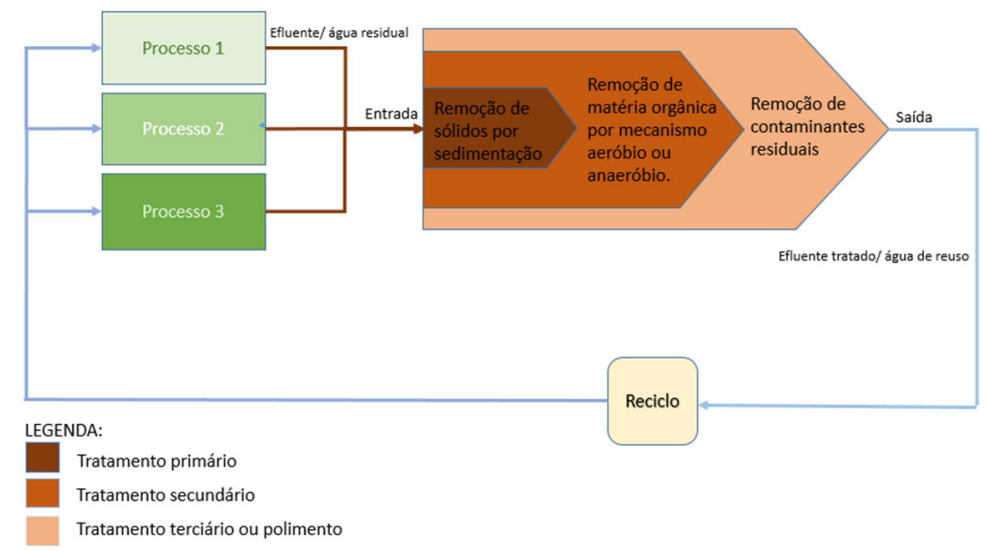

Fonte: Própria, 2019

\section{CONCLUSÃO}

As pesquisas sobre redes de reuso de águas ganhou grande destaque nos últimos anos, com um aumento considerável no número de artigos publicados. A partir da pesquisa sistemática foi possível identificar informações sobre o estado da arte do que está sendo pesquisado, o que deve ser levado em conta e os aspectos relevantes na implantação de uma rede de reuso de água.

Uma atenção especial foi dada aos trabalhos de Alnouri, et al ${ }^{[3,5,9]}$, onde são levantados aspectos como a topologia local (corredores de acesso, barreiras dentro 
das plantas, barreiras naturais e elevações), o tratamento centralizado ou descentralizado dos efluentes e a mistura das correntes de contaminante para tratamento. Além disso, conceitos como o de eco parques industriais, que compartilham recursos são explorados no contexto das redes uma vez que os efluentes produzidos por diferentes empresas dentro de uma mesma localidade podem ser tratados e redistribuídos dentro, das unidades industriais, de acordo com a vazão e com a qualidade requerida para cada um dos usos.

\section{REFERÊNCIAS}

${ }^{1}$ MIERZWA, J. C; HESPANHOL, I. Água na indústria: uso racional e reuso [1 ${ }^{a}$ reimpr.]- São Paulo, oficina dos textos, 2017.

2 NETO, G. C. O; et al. Princípios e ferramentas da produção mais limpa: um estudo exploratório em empresas brasileiras. 2015.

${ }^{3}$ ALNOURI, S. Y.; LINKE, P.; EL-HALWAGI, M. M. A synthesis approach for industrial city water reuse networks considering central and distributed treatment systems. 2014.

${ }^{4}$ Fadzil, A.F.A; Alwi, S.R.W; Manan, Z; Klemas, J.J. Industrial site water minimisation via one-way centralised water reuse reader, 2018

${ }^{4}$ MIRRE, R. C.; FERREIRA, S. C. L.; DIAS, A. R.; PESSOA, F.L.P. Conservação e reuso de águas usando o método diagrama de fontes de água para processos em batelada: um estudo de caso. 2012

${ }^{5}$ ALNOURI, S. Y.; LINKE, P.; EL-HALWAGI, M. M. Synthesis of industrial park water reuse networks considering treatment systems and merged connectivity options. 2016

6 CONFEDERAÇÃO NACIONAL DA INDÚSTRIA (CNI). Reuso de efluentes: metodologia para análise do potencial do uso de efluentes tratados para abastecimento industrial- Brasília: 2017

8 TAN, R.R; AVISO, K.B; CRUZ JR. A.B; CULABA, A.B. A note on an extended fuzzy bi-level optimization approach for water exchange in eco-industrial parks with hub topology. 2011

${ }^{9}$ ALNOURI, S.Y; LINKE, P; EL-HALWAGI, M. Water integration in industrial zones: a spatial representation with direct recycle applications. 2015

10 SUÁREZ, R.H; MATA, J.M.Z; GUEVARA, F.M; LEYTE, R.L. Análisys y reconfiguración de líneas de efluentes a una unidad de tratamiento multicontaminante. $-2008$.

${ }^{12}$ Wang Y. P, Smith R. Design of distributed effluent treatment systems. 1994

${ }^{13}$ LI, A.H; YANG, Y.Z; LIU, Z.Y. A numerical-indicator-based method for design of distributed wastewater treatment systems with multiple contaminants. 2015 